\title{
BLACK HOLES: THE LEGACY OF HILBERT'S ERROR
}

\author{
LEONARD S. ABRAMS
}

\begin{abstract}
The historical postulates for the point mass are shown to be satisfied by an infinity of space-times, differing as to the limiting acceleration of a radially approaching test particle. Taking this limit to be infinite gives Schwarzschild's result, which for a point mass at $x=y=z=0$ has $C(0+)=\alpha^{2}$, where $\alpha=2 m$ and $C(r)$ denotes the coefficient of the angular terms in the polar form of the metric. Hilbert's derivation used the variable $r^{*}=[C(r)]^{1 / 2}$, which transforms the coordinate location of the point mass to $r_{0}^{*}=[C(0+)]^{1 / 2}$. For Hilbert, however, $C$ was unknown, and thus could not be used to determine $r_{0}^{*}$. Instead he asserted, in effect, that $r^{*}=\left(x^{2}+y^{2}+z^{2}\right)^{1 / 2}$, which places the point mass at $r^{*}=0$. Unfortunately, this differs from the value $(\alpha)$ obtained by substituting Schwarzschild's $C$ into the expression for $r_{0}^{*}$, and since $C(0+)$ is a scalar invariant, it follows that Hilbert's assertion is invalid. Owing to this error, in each spatial section of Hilbert's spacetime, the boundary $\left(r^{*}=\alpha\right)$ corresponding to $r=0$ is no longer a point, but a two-sphere. This renders his space-time analytically extendible, and as shown by Kruskal and Fronsdal, its maximal extension contains a black hole. Thus the Kruskal-Fronsdal black hole is merely an artifact of Hilbert's error.
\end{abstract}

Il existe un nombre infini d'espace-temps non équivalents pour la masse punctuelle; ils diffèrent les unes des autres quant à l'accélération limite d' une particule d'essai s'approchant radialement. En faisant cette limite infinie, on a l'espace-temps inextensible de Schwarzschild, qui a, pour une masse punctuelle à $x=y=z=0, C(0+)=\alpha^{2}$, ou $\alpha=2 m$ et $C(r)$ désigne le coefficient des termes angulaires lorsque la métrique est écrite en polaires sphériques. Hilbert utilisait dans sa dérivation la variable $r^{*}=[C(r)]^{1 / 2}$, qui transforme la position de la masse punctuelle de $r_{0}^{*}=0$ à $r_{0}^{*}=[C(0+)]^{1 / 2}$. Pour Hilbert cependant, $C$ était une inconnue, et il ne pouvait par conséquent l'utiliser pour determiner $r_{0}^{*}$. Au lieu de cela, il affirmait en effet que $r^{*}=\left(x^{2}+y^{2}+z^{2}\right)^{1 / 2}$, ce qui place la masse punctuelle à $r^{*}=0$. Malhereusement, cette valeur diffère de la valeur $(\alpha)$ obtenue en substituant le $C$ de Schwarzschild dans l'expression de $r_{0}^{*}$; comme $C(0+)$ est une scalaire invariant, il s'ensuit que l'affirmation de Hilbert est invalide. Comme résultat, dans chaque section spatiale de l'espace-temps de Hilbert, la limite $\left(r^{*}=\alpha\right)$ correspondant à $r=0$ n'est plus un point mais une sphère bidimensionnelle et par conséquent pas une singularité quasi régulière. Cela rend son espace-temps analytiquement extensible, et, comme l'ont montré Kruskal et Fronsdal, son extension maximale contient un trou noir. Le trou noir Kruskal-Fronsdal n'est donc rien de plus qu'un produit de l' erreur de Hilbert.

Published in Can. J. Phys. 67 (1989) 919.

Received November 10, 1988. 


\section{INTRODUCTION}

Ever since Schwarzschild's 1916 derivation [1], it has been accepted that the historical postulates used by him to characterize the point-mass spacetime do, in fact, lead to a unique gravitational field. However, Schwarzschild's result was obtained years before the concept of a 'quasiregular' singularity [2] had surfaced. As shown here, when the general polar form of a static, spherically symmetric metric

$$
g_{U}(r)=A(r) d t^{2}-B(r) d r^{2}-C(r) d \Omega^{2}
$$

is substituted into Einstein's vacuum field equations and the remaining historical postulates are imposed on the solution, there results a one-parameter family of inequivalent, maximal space-times, each of which has a quasiregular singularity at the location of the point mass. Since each of these space-times assigns a different number to the limiting value of a radially approaching test particle's locally measured acceleration, it is necessary to supplement the historical postulates by one that fixes this limit. Taking it to be infinite gives Schwarzschild's result

$$
g_{S}(r)=(1-\alpha / R) d t^{2}-(1-\alpha / R)^{-1} d R^{2}-R^{2} d \Omega^{2}
$$

where $\alpha=2 m$ and $R=\left(r^{3}+\alpha^{3}\right)^{1 / 3}$.

Several months after Schwarzschild's paper was published, another derivation of the point-mass space-time was given by Hilbert [3]. At the start he introduced the radial coordinate $r^{*}=[C(r)]^{1 / 2}$, and then solved the vacuum field equations for the two remaining unknowns, obtaining:

$$
g_{H}\left(r^{*}\right)=\left(1-\alpha / r^{*}\right) d t^{2}-\left(1-\alpha / r^{*}\right)^{-1} d r^{* 2}-r^{* 2} d \Omega^{2}
$$

Of course, the use of $r^{*}=[C(r)]^{1 / 2}$ transforms the coordinate location of the point mass from $r=0$ to $r^{*}=[C(0+)]^{1 / 2}$. As can be seen from Schwarzschild's result, this is simply $\alpha$. Hilbert, however, claimed that $r^{*}=\left(x^{2}+y^{2}+z^{2}\right)^{1 / 2}$, so that the point mass represented by $g_{H}\left(r^{*}\right)$ was at $r^{*}=0$, and this claim went unchallenged by his contemporaries. As a result of this mistake, in each spatial section the locus $r^{*}=\alpha$ is not a point but a two-sphere, and thus no longer constitutes a quasiregular singularity of Hilbert's space-time $S_{H}$. As shown by Kruskal [4] and Fronsdal [5], with $r^{*}=\alpha$ having the character of a two-sphere in the $t=$ constant hypersurfaces, $S_{H}$ is analytically extendible to $r^{*}>0$, and the so-extended space-time contains a black hole. It follows that the theoretical foundation of spherical black holes is based on the 1916 error of Hilbert.

It is the principal objective of this paper to show how Hilbert came to make this mistake. It will also be shown that $S_{H}$ cannot be used to represent the 'exterior' of a collapsing star, and thus that spherical black holes cannot be produced by gravitational collapse. 


\section{The SPACE-TIME OF A POINT MASS}

Consider any static, time-symmetric and spherically symmetric configuration $(U)$ of matter and (or) energy. In his paper [1] Schwarzschild regarded it as self evident that the metric of such a configuration, when expressed in terms of quasi-Cartesian coordinates $(t, x, y$, and $z)$, was necessarily of the form

$$
\begin{gathered}
g_{U}(x, y, z \mid 0,0,0)=F(r) d t^{2}-G(r)\left(d x^{2}+d y^{2}+d z^{2}\right) \\
-H(r)(x d x+y d y+z d z)^{2}
\end{gathered}
$$

where

$$
r=\left(x^{2}+y^{2}+z^{2}\right)^{1 / 2}
$$

and

$$
F, G, H>0
$$

and where, without loss of generality, the center 19 of the spherical symmetry has been taken at $x=y=z=0$ for all $t$; this is signified by the notation ' $0,0,0$ ' in the argument of $g_{U}$. (The generality of (2) for such configurations was subsequently established by Eiesland [7].)

In polar coordinates $(r, \theta$, and $\phi)$, where $x=r \sin \theta \cos \phi$, etc., the above expression of $g_{U}$ becomes

$$
g_{U}(r \mid 0)=A(r) d t^{2}-B(r) d r^{2}-C(r) d \Omega^{2}
$$

where

$$
d \Omega^{2}=d \theta^{2}+d \phi^{2} \sin ^{2} \theta
$$

and from (4) and the tensor transformation law, it follows that

$$
A, B, C>0
$$

(Analogously, the ' 0 ' in the argument of $g_{U}$ in (5) signifies that the center of symmetry of (5) is at $r=0$ for all $t$.)

For later purposes, we note that apart from spatial rotations about the origin, which are of no relevance to what follows, the only transformations $T$ that leave the structure of (5) unchanged are of the form

$$
\begin{array}{cc}
t=k \bar{t}+b, & k \neq 0, b \text { constants } \\
r=h(\bar{r}), & h \in C^{1}
\end{array}
$$

Since $C(r)$ in (5) is readily seen to be a scalar under $T$, it follows that:

\footnotetext{
${ }^{1}$ Since a point mass is the only configuration under consideration, it will be assumed here that only a single center of symmetry is present.

${ }^{2}$ The center is that three-dimensional point having the property that rotations about it leave the Newtonian description of $U$ unchanged. Note that the very concept of spherical symmetry presupposes that the center of symmetry is a three-dimensional point in each spatial section [6].
} 
THEOREM:The value of $C$ at a specified event $P$ (this will be denoted by ' $\left.C[P]^{\prime}\right)$ is an invariant of the space-time associated with a given $U$.

Consequently, two space-times $S_{1}$ and $S_{2}$ having metrics of the form (5) for which $C_{1}[P] \neq C_{2}[P]$ are necessarily inequivalent. A fortiori,

COROLLARY:If two space-times $S_{1}$ and $S_{2}$ have metrics of the form (5), and if $\lim C_{1}[P] \neq \lim C_{2}[P]$ as $P$ approaches the center of symmetry, then $S_{1}$ and $S_{2}$ are inequivalent.

Consider now the particular $U$ consisting of a single uncharged, nonrotating, nonradiating point mass (whose Newtonian gravitational mass will henceforth be denoted by ' $m$ '). Historically, the conditions regarded as distinguishing the space-time $\left(M_{U}, g_{U}\right)$ of this $U$ from those of all others where originally formulated by Einstein [8], and together with those implicit in that formulation were enumerated by Finkelstein [9]. The expression on the right-hand side of (5), together with (16) and (7), already satisfies the static- and spherical-symmetry requirements of Finkelstein's list, as well as those requiring a Lorentz signature and a global-time coordinate. As shown in Appendix A, the further requirements that (5) be analytic, satisfy the vacuum field equations, and be asymptotically flat reduce (5) to

$$
g_{P M}(r \mid 0)=\left(1-\alpha / C^{1 / 2}\right) d t^{2}-\frac{C^{\prime 2}}{4 C\left(1-\alpha / C^{1 / 2}\right)} d r^{2}-C d \Omega^{2}
$$

where $C(r)$ is any analytic function of the $r$ in (3) having the following three properties:

$$
\begin{array}{r}
C(0+) \equiv \beta^{2} \geq \alpha^{2} \\
C^{\prime}(r)>0, r>0 \\
C(r) / r^{2} \rightarrow 1 \text { as } r \rightarrow \infty
\end{array}
$$

(the prime denotes differentiation with respect to $r$ ). As shown there, all such metrics comply with the final requirement of being analytically inextendible to $r=0$.

Since the center of symmetry of (5) is located at $r=0$, the same is true of the center of symmetry of (10), whence it follows from the corollary that two space-times defined on $M_{0}: r>0$ and having $g_{P M}$ as their metric are inequivalent if $C_{1}(0+) \neq C_{2}(0+)$. This, together with (11), shows that there is a one-parameter family of inequivalent space-times (distinguished by their value of $C(0+)$ ), which satisfy all the historical postulates for the point mass. That is: 
The historical postulates for the point mass do not result in a unique space-time. To obtain such a space-time, it is necessary to supplement those postulates by one which fixes the value of $C(0+) \cdot$.

To determine this supplementary postulate, it is necessary to relate $C(0+)$ to some physical property of the point mass. To this end, consider the motion of an uncharged, nonspinning, nonradiating test particle as it approaches the point mass along a radial geodesic. As shown by Doughty [13], the particle "locally measured" acceleration (i.e., its acceleration measured by a nearby observer whose position is fixed with respect to the point mass (see Appendix B) is given by:

$$
a=\frac{\left(-g_{r r}\right)^{1 / 2}\left(-g^{r r}\right)\left|g_{00, r}\right|}{2 g_{00}}=\frac{\alpha}{2 C\left(1-\alpha / C^{1 / 2}\right)^{1 / 2}}
$$

which approaches

$$
a_{0}=\frac{\alpha}{2 \beta^{3 / 2}(\beta-\alpha)^{1 / 2}}
$$

as $r \rightarrow 0$. Consequently, for a given value of $m$, the value of $C(0+)$ is determined by the limiting value of a test-particle's locally measured acceleration as it approaches the point mass along a radial path.

Since there are no experimental data concerning $a_{0}$, it is necessary to choose its value on the basis of theoretical arguments. One such argument is that in the corresponding Newtonian situation $a_{0}$ is infinite. Although one would not expect strict quantitative agreement (in the sense of equal values of $a$ at equal values of proper distance), the fact that the curvature invariant $f=R_{i j k m} R^{i j k m}$ remains finite as $r \rightarrow 0$ indicates that the relativistic case differs from the Newtonian one only in degree, not in kind. Accordingly, since there are no "degrees" associated with an infinite limit, the supplementary postulate is taken to be:

(i) the limiting value of a neutral nonspinning nonradiating test particle's locally measured acceleration as it approaches the point mass along a radial geodesic is infinite.

Examination of (15) shows that the only values of $\beta$ that make $a_{0}$ infinite are 0 and $\alpha$, and of these only the latter satisfies (11). Hence, the result of postulate $(i)$ is

$$
C(0+)=\alpha^{2}
$$

The simplest choice of analytic $C$ satisfying (12), (13), and (16) is

$$
C_{B}(r)=(r+\alpha)^{2}
$$

\footnotetext{
${ }^{3}$ The fact that a boundary condition for $r \rightarrow 0$ is just as necessary as the one for $r \rightarrow \infty$ was first realized by Brillouin [10]. Although Abrams' work (see ref. [11]) contained a derivation of the general point-mass metric similar to that given here in Appendix A, it made use of an invalid argument (see ref. [12]) to prove that $C(0+)=\alpha^{2}$, and thus did not turn up the fact that an additional postulate was required.
} 
which when substituted into (10) results in the following form, first used by Brillouin [14], for the metric of a point mass at $r=0$ :

$$
g_{B}(r \mid 0)=\frac{r}{r+\alpha} d t^{2}-\frac{r+\alpha}{r} d r^{2}-(r+\alpha)^{2} d \Omega^{2}
$$

The metric obtained by Schwarzschild corresponds to the choice

$$
C_{S}(r)=\left(r^{3}+\alpha^{3}\right)^{2 / 3}
$$

which satisfies (12), (13), and (16) by inspection.

It should be noted that all space-times $\left[M_{0}, g_{P M}(r \mid 0)\right]$ arising from (10) via an analytic $C$ satisfying (12), (13), and (16) are equivalent, being related via $C^{\omega}$ global diffeomorphisms of the form

$$
C_{i}(r)=C_{j}(\bar{r})
$$

and being globally homeomorphic and maximal, as well as having the same singularity at $r=\bar{r}=0$. Consequently, the space-time $S_{S}=\left[M_{0}, g_{B}(r \mid 0)\right]$ will henceforth be termed "Schwarzschild's", since it is equivalent to the $\left[M_{0}, g_{S}(r \mid 0)\right]$ actually obtained by him, and its use simplifies the subsequent discussion.

\section{Transformation to Flamm's Form}

Let us relabel the events of $M_{0}$ by adding $\alpha$ to their $r$ values, and denote the new "radial" coordinate by $\bar{r}$; then

$$
\bar{r}=r+\alpha, r>0
$$

This transforms $M_{0}$ to $\bar{M}_{\alpha}$, where

$$
\bar{M}_{\alpha}: \bar{r}>\alpha
$$

tranforms the location of the point mass at $r=0$ to $\bar{r}=\alpha$, and transforms $g_{B}(r \mid 0)$ to

$$
g_{F}(\bar{r} \mid \alpha)=\frac{\bar{r}-\alpha}{\bar{r}} d t^{2}-\frac{\bar{r}}{\bar{r}-\alpha} d \bar{r}^{2}-\bar{r}^{2} d \Omega^{2}
$$

termed the Flamm metric since Flamm 15] was the first to use this form to represent a point mass at $r=\alpha$.

(To avoid misunderstanding, note that the statement: "The point mass is at $r=0$," in connection with (18), and "The point mass is at $\bar{r}=\alpha$ ", in connection with (23), do not mean that these $r$ or $\bar{r}$ values are welldefined quantities, let alone that events having such values are part of the associated space-times. What they do mean is that, in the case of (18), the proper distance from the point mass to an event in $M_{0}$ with coordinate $r$ tends to 0 as $r \rightarrow 0$; and in the case of (23), that the proper distance from the point mass to an event in $\bar{M}_{\alpha}$ with coordinate $\bar{r}$ tends to 0 as $\bar{r} \downarrow \alpha$.)

Since physics is not changed by such a relabelling, it follows that the Flamm space-time:

$$
S_{F}=\left[\bar{M}_{\alpha}, g_{F}(\bar{r} \mid \alpha)\right]
$$


represents the identical physical situation as $S_{S}$, namely, a single uncharged, nonrotating, nonradiating point mass. Thus, both $S_{S}$ and $S_{F}$ have the same singularity structure, i.e., a quasiregular singularity at the location of the point mass, and thus $S_{F}$ is likewise analytically inextendible to that location, now denoted by $\bar{r}=\alpha$.

In passing, note that the fact that the coefficient of $d \Omega^{2}$ in (23) tends to 0 as $\bar{r} \downarrow 0$ does not contradict (11), since the $\bar{r}$ in (23) is not the $r$ in (3).

\section{Hilbert's DERIVATION OF THE POINT-MASS METRIC}

About 10 months after the publication of ref. [1], Hilbert [3] presented another derivation of the point-mass metric. Although Hilbert's starting point was the same expression (5) used by Schwarzschild, he immediately reduced the number of unknowns to two by introducing a new "radial" coordinate $r^{*}$, defined by

$$
r^{*}=[C(r)]^{1 / 2}
$$

This transforms (5) to

$$
g_{U}^{*}\left(r^{*} \mid 0^{*}\right)=A^{*}\left(r^{*}\right) d t^{2}-B^{*}\left(r^{*}\right) d r^{* 2}-r^{* 2} d \Omega^{2}
$$

where from (7)

$$
A^{*}, B^{*}>0
$$

and $0^{*}$ denotes the value of $r^{*}$ at the location of the point mass, which from (25) is given by

$$
0^{*}=[C(0+)]^{1 / 2}
$$

Solving the resulting vacuum field equations by means of a variational principle, Hilbert arrived at the following expression for the point-mass metric:

$$
g_{F}\left(r^{*} \mid 0^{*}\right)=\frac{r^{*}-\alpha}{r^{*}} d t^{2}-\frac{r^{*}}{r^{*}-\alpha} d r^{* 2}-r^{* 2} d \Omega^{2}
$$

which by inspection is well defined on $M_{\alpha}^{*}: r^{*}>\alpha$.

However, there are two problems connected with the use of (25), which Hilbert evidently overlooked. The first of these is that (25) involves a loss of generality [16]; for example, if $U$ is such that the $C$ in (1) is constant [17], then the use of (25) will make it impossible to determine the metric for $U$. Unfortunately (from the stand-point of subsequent developments), this flaw had no impact on Hilbert's derivation, since as seen in Sect. 2, for the point-mass metric all $C(r)$ permissible in (5) are strictly monotonic, so that (25) is in fact a diffeomorphism for such a $U$.

The second is that the use of (25) destroys information; once it is applied, it becomes impossible to determine the relationship between $r^{*}$ and $r$ (and thus to find the value of $\left.0^{*}\right)$, since at this point in the derivation the function $C(r)$ in (25) is unknown, and there is no way to determine what it is (or even its value as $r \rightarrow 0$ ) from the resulting $A^{*}$ and $B^{*}$. 
This being the case, how did Hilbert arrive at the value of $0^{*}$ ? The answer is as follows: by assuming that for all $U$, the triplet $\left(r^{*}, \theta\right.$, and $\left.\phi\right)$ can be regarded as polar coordinates just as validly as $(r, \theta$, and $\phi)$, so that $r^{*}=\left(x^{2}+y^{2}+z^{2}\right)^{1 / 2}$. From this assumption, together with the fact that the location of the point mass described by (5) is given by $x=y=z=0$, it follows at once that even without knowing $C(r)$, the $r^{*}$ position of the point mass described by (26) is given by $r^{*}=0$, and so Hilbert proclaimed.

While it is true that there are a number of $U$ for which Hilbert's assumption is valid, the point mass is not one of them, as shown in Sect. 2. For such a $U$ all $C$ in (5), and thus in (25), have the property that $C(0+)=\alpha^{2}$, whence (28) shows that $0^{*}=\alpha>0$. Thus, as the notation on the left-hand side of (29) indicates, the right-hand side is simply Flamm's metric for a point mass at $r^{*}=\alpha$.

Henceforth, to avoid circumlocution the right-hand side of (29) together with the assumption that the $r^{*}$ appearing therein is related to the $x, y$, and $z$ in (2) via (3), and thus that the point mass described by (29) is at $r^{*}=0$, will be termed "Hilbert's" metric 1 , and denoted by $g_{H}$ :

$$
g_{H}\left(r^{*} \mid 0\right)=\frac{r^{*}-\alpha}{r^{*}} d t^{2}-\frac{r^{*}}{r^{*}-\alpha} d r^{* 2}-r^{* 2} d \Omega^{2}
$$

while Hilbert's space-time will be denoted by $S_{H}$

$$
S_{H}=\left[M_{\alpha}^{*}, g_{H}\left(r^{*} \mid 0\right)\right]
$$

\section{Extendibility of HilberT's SPACE-Time}

Consider the circle $\gamma(\epsilon)$ mentioned at the end of Appendix A. In $S_{S}$, its description is given by $t=t_{0}, \theta=\pi / 2$, and $r=\epsilon$, where $t_{0}$ is an arbitrary constant, independent of $\epsilon$. Since $S_{S}$ is diffeomorphic to $S_{H}$ via $T_{\alpha}: M_{0} \rightarrow M_{\alpha}^{*}$ by $r^{*}=r+\alpha$, the description of $\gamma(\epsilon)$ in $S_{H}$ can be obtained by applying $T_{\alpha}$ to its description in $S_{S}$. This gives $t=t_{0}, \theta=\pi / 2$, and $r^{*}=\epsilon+\alpha$. As seen from (30), its proper circumference is still $2 \pi(\epsilon+\alpha)$, which as in Appendix A tends to $2 \pi \alpha$ as $\epsilon \downarrow 0$. Now, however, in contrast to the situation in Appendix A, this no longer gives rise to a violation of elementary flatness at $T_{\alpha}(0)=\alpha$ in $S_{H}$ because, thanks to the assumption that gave birth to (30), $\gamma(\epsilon)$ no longer shrinks down to a point as $\epsilon \downarrow 0$, but instead simply approaches the circle $r^{*}=\alpha$. Thus, Hilbert's erroneous (for a point mass) assumption results in the disappearance of the quasiregular singularity that is present in Flamm's space-time at $\bar{r}=\alpha$. Since it is well known that there are no curvature-type singularities of $g_{H}$ at $r^{*}=\alpha$,

\footnotetext{
${ }^{4}$ In ref. 11], this metric was designated "Droste-Weyl". The reason was that both Droste [18] in 1916 and Weyl [19] in 1917 had derived metrics having the form of $g_{H}$, whereas my copy of Hilbert's paper was dated 1924, so that it appeared that Droste and Weyl had been the first to obtain $g_{H}$. However, I subsequently discovered that Hilbert's paper had originally been published in 1916, and that neither Droste nor Weyl had claimed that the $r$ coordinate appearing in their metrics was $\left(x^{2}+y^{2}+z^{2}\right)^{1 / 2}$. Thus, Hilbert alone was responsible for the error.
} 
it follows that there are no singularities of any kind there, so that $S_{H}$ is analytically extendible to $r^{*}=\alpha$, and as Kruskal and Fronsdal have shown, all the way to $r^{*}>0$.

\section{Inequivalence of Schwarzschild and Hilbert SPACE-Times}

If two space-times are to be equivalent, it is certainly necessary that they be isometric i.e., that there exists a diffeomorphism from one to the other that carries the metric of one into the metric of the other. And since the presence of singularities of the manifold geometry is unaffected by diffeomorphisms, it is also necessary that equivalent space-times have the same "singularity structure", i.e., the same singularities as one approaches corresponding boundary points. Now, $S_{S}$ and $S_{H}$ are isometric under $T_{\alpha}$, but as shown in the preceding section, $S_{H}$ has no singularity corresponding to the quasiregular singularity at $r=0$ in $S_{S}$. Consequently, $S_{S}$ and $S_{H}$ are inequivalent. Since it was shown in Sect. 2 that $S_{S}$ is the space-time of a point mass, it follows that $S_{H}$ and its analytic extension $\left(S_{K-F}\right)$ are not.

\section{The Kruskal-Fronsdal Black hole is unneCessary}

Consider any phenomenon supposedly involving a single Kruskal-Fronsdal (K-F) black hole (e.g., x-ray spectra from accreting gas). Because of the infinite red shift at the surface $\left(r^{*}=\alpha\right)$ of the hole, all that we can ever know of this phenomenon must arise from information originating outside the hole. But the space-time "exterior" to the hole is $S_{H}$, which in turn is diffeomorphic to $S_{S}$. That is to say, everything that takes place outside the hole would occur in the identical fashion if the entire space-time $\left(S_{K-F}\right)$ were replaced by $S_{S}$; it is impossible to determine which space-time is "really" present. Thus, any observations that are explicable by postulating the presence of a K-F black hole are equally well explained by postulating the presence of a Schwarzschild point mass at the geometrical center of the black-hole's surface. Consequently, there is no need to involve a K-F black hole to explain any set of observations, Schwarzschild's "black point" will do an equally effective job.

Whether this observational equivalence extends to the case of two or more black holes (vis-a-vis two or more point masses) is unclear, but in view of the remarks of the next two sections it does not seem worthwhile to pursue the matter.

\section{The K-F Black Hole IS UnProducible}

The valid proofs of Birkhoff's theorem (e.g. refs. [7] and [20]) show only that any spherically symmetric solution of the vacuum-field equations can be given the form of $g_{H}$, but say nothing as to the relationship of the radial coordinate of the transformed metric and the underlying quasi-Cartesian $x$, $y$, and $z$. As emphasized by Brans [21], until this relationship is known the metric is undefined. Thus, Birkhoff's theorem cannot be used to justify the 
claim that the metric exterior to a spherically symmetric star is identical to that of a point mass.

However, the characteristics of the space-time exterior to a spherically symmetric, uncharged, nonrotating, nonradiating star are nearly the same as those of a point mass, the only difference being that for the star the postulates relating to the behavior of the space-time at $r=0$ are no longer applicable. Consequently, to determine the exterior metric for such a star all that is necessary is to impose the remaining postulates for the point mass on (5). As can be seen by inspection of Appendix A, doing so changes nothing up through (A.16), while (A.17) must be replaced by

$$
C\left(r_{b}\right) \geq \alpha^{2}
$$

where $r_{b}$ denotes the $r$ coordinate of the star's boundary. (The precise value of $C\left(r_{b}\right)$ consistent with (32) is determined by the junction conditions.) Consequently, the exterior metric of such a star is given by $g_{P M}(r \mid 0)$ in (10), but with $C$ now satisfying (12) for $r>r_{b}$, (13), and (32). That is to say, the exterior metric has the same functional form as the metric of a point mass located at the star's center, but the values of the parameters appearing therein are different. An example of such a situation can be found in ref. [22].

In the case of collapse to a point, this distinction ultimately vanishes, so that the appropriate space-time for the exterior of a star undergoing catastrophic collapse tends to the $\left(S_{S}\right)$ of a point mass. While the precise

details of the approach to $S_{S}$ will vary from case to case, it is already clear from the form of $g_{P M}$, together with (13) and (32), that $A(r)>0$ for all $r>r_{b}$, and thus, that no black hole ever forms in the exterior of the star, no matter how far the collapse proceeds.

Thus, the correction of Hilbert's error not only eliminates the point mass as a possible source of K-F black holes, but simultaneously deprives them of the only mechanism for their production.

\section{The K-F Black hole is unreal}

Although it was shown in Sect. 6 that $S_{K-F}$ does not represent the spacetime of a point mass, it might still be hoped that it represents some other configuration of matter and (or) energy, and is thus of physical significance in its own right.

However, this is not the case. Since the energy-momentum tensor vanishes everywhere in $S_{K-F}$, the only possible locations of its sources are at its singularities. These are at $r^{*}=0$, or in terms of Kruskal's $u$ and $v$, at $v^{2}-u^{2}=1$. As is easily seen, these loci are spacelike, whereas those of real matter or radiation are timelike or null, respectively. Consequently, it is impossible for $S_{K-F}$ to represent any real configuration of matter and (or) energy, i.e., $S_{K-F}$ is physically unreal, and thus so is the K-F black hole.

While this disposes of the reality of $S_{K-F}, S_{K-F}$ possesses another property worth mentioning in connection with other types of black holes, namely, 
the presence at $r^{*}=\alpha$ of an interior "surface of infinite acceleration" (at which the locally measured acceleration of test particles becomes infinite: see ref. 23]), despite the fact that there are neither matter singularities nor geometrical singularities at that surface. This existence of such a surface interior to the space-time is a direct result of Hilbert's assumption regarding $r^{*}$, since it was this that transferred the "Cheshire cat" (the point mass) to a distant point, while leaving the "grin" (the infinite acceleration of test particles) intact at $r^{*}=\alpha$.

\section{Conclusions}

We summarize the result of the preceding sections as follows. The K-F black hole is the result of a mathematically invalid assumption, explains nothing that is not equally well explained by $S_{S}$, cannot be generated by any known process, and is physically unreal. Clearly, it is time to relegate it to the same museum that holds the phlogiston theory of heat, the flat earth, and other will-o'-the-wisps of physics.

Consider next the family $(\mathcal{S})$ of black holes obtained by analytic extension of metrics, which, for certain values $\left(\beta_{0}\right)$, of their parameters $(\beta)$, reduce to $g_{H}$ (e.g., Reissner-Nordström, Kerr, Kerr-Newman, etc.). Some, like Reissner-Nordström's solution for the point charge, were likewise derived from a set of postulates characterizing the specified matter and (or) energy configuration, and thus their derivations must be analyzed to determine whether an error such as that made by Hilbert was committed. Others were simply "discovered", and their sources sought afterwards, so it is impossible to determine whether they are based on an invalid assumption. However, the fact that all such metrics are regarded as reducing to $g_{H}$ when $\beta=\beta_{0}$ (see ref. [24], 25], and [26]) shows that it is tacitly assumed that the radial coordinate appearing in these metrics is equal to $\left(x^{2}+y^{2}+z^{2}\right)^{1 / 2}$, and Sect. 4 shows that this assumption is invalid when $\beta=\beta_{0}$. Moreover, each of the associated black-hole spacetimes bears the telltale stigma (an interior surface of infinite acceleration, see ref. [27]) associated with the transfer of boundary behavior to interior events that was shown in the previous section to be a consequence of precisely that assumption for the case of K-F black holes. Accordingly, members of $\mathcal{S}$ are highly suspect.

Finally, there remain those black holes that have likewise simply been discovered, but whose exterior metrics do not reduce to $g_{H}$ for any values of their parameters (e.g., the "toroidal" black holes described in ref [28]). Their status awaits an investigation of the reasonableness of their sources.

\footnotetext{
${ }^{5} \mathrm{~A}$ paper proving that this is indeed the case for the point-charge metric is shortly to be submitted by the author.
} 


\section{ACKNOWLEDGMENTS}

It is a pleasure to acknowledge a number of helpful conversations with B. O'Neill and R. Greene, and with M. Morris, as well as the critiques of the author's earlier paper on this subject by R. Gautreau and C. Will.

\section{ERRATUM [33}

Section 6 should be replaced by the following paragraph:

\section{Inequivalence of Schwarzschild and Hilbert universes}

By inspection, $S_{S}$ and $S_{H}$ are isometric via $T_{\alpha}$ and thus equivalent. However, it was shown above that due to the difference in the topology of their boundaries, they are associated with different singularity structures. Thus, the universes $\left(U_{S}\right.$ and $\left.U_{H}\right)$ corresponding to $S_{S}$ and $S_{H}$ (with their indicated boundaries) are inequivalent (cf. Abrams, L.S., Physica A, 227 (1996) 131). Since it follows from Sect. 2 that $U_{S}$ is the universe of a point-mass, then a fortiori $U_{H}$ is not. For the same reason, this last is also true of $U_{K F}$, the universe corresponding to the maximal analytic extension $\left(S_{K F}\right)$ of $S_{H}$ found by Kruskal and Fronsdal.

Section 7, line 12 should read: black hole's "surface".

\section{REFERENCES}

1. Schwarzschild, K., Sitzungsber. Preuss. Akad. Wiss., Phys. Math. Kl. 1916, 189.

2. Ellis, G.F.R., and Schmidt, B., Gen. Rel. Gravitation 8 (1977) 915.

3. Hilbert, D., Nachr. Ges. Wiss. Göttingen, Math. Phys. Kl. 1917, 53. (Read 23 Dec. 1916).

4. Kruskal, M., Phys. Rev. 119 (1960) 1743.

5. Fronsdal, C., Phys. Rev. 116 (1959) 780.

6. Demianski, M., Relativistic astrophysics, Pergamon Press, New York (1985) p. 6.

7. Eiesland, J., Trans. Am. Math. Soc. 27 (1925) 213.

8. Einstein, A., Sitzungsber. Preuss. Akad. Wiss., Phys. Math. Kl. 1915, 831.

9. Finkelstein, D., Phys. Rev. 110 (1958) 965.

10. Brillouin, L., Relativity reexamined, Academic Press, New York (1970) p. 87.

11. Abrams, L.S., Phys. Rev. D 20 (1979) 2474.

12. Abrams, L.S., Phys. Rev. D 21 (1980) 21.

13. Doughty, N., Am. J. Phys. 49 (1981) 412.

14. Brillouin, M., C. R. Seances Acad. Sci., Ser. A 175 (1922) 1009.

15. Flamm, L., Phys. Z. 17 (1916) 448.

16. Krasinski, A. and Plebanski, J., Rep. Math. Phys. 17 (1980) 217.

17. Nariai, H., Sci. Rep. Tohoku Imp. Univ., Ser. 124 (1950) 160.

18. Droste, J., Proc. K. Ned. Akad. Wet., Ser. A 19 (1917) 197. (Read May 1916).

19. Weyl, H., Ann. Phys. (Leipzig) 54 (1917) 117.

20. Bonnor, W.B., in Recent developments in general relativity, Pergamon Press, New York (1962) p. 167.

21. Brans, C., Phys. Rev. 110 (1965) B1174. 
22. Schwarzschild, K., Sitzungsber. Preuss. Akad. Wiss., Phys. Math. Kl. 1916, 424.

23. Rindler, W., Phys. Rev. 119 (1960) 2082.

24. Hernandez Jr., W., Phys. Rev. 167 (1968) 1180.

25. Tominatsu, A. and Sato, H., Phys. Rev. Lett. 29 (1972) 1344.

26. Lightman, A., Press, W., Price, R., and Teukolsky, S., Problem book in relativity and gravitation, Princeton University Press, Princeton, NJ. (1975) p. 101.

27. Doughty, N., Am. J. Phys. 49 (1981) 720.

28. Geroch, R. and Hartle, J.B., J. Math. Phys. (N.Y.) 23 (1982) 680.

29. Tolman, R.C., Relativity, thermodynamics and cosmology, Oxford University Press, London (1934) pp. 265-266.

30. Tolman, R.C., Relativity, thermodynamics and cosmology, Oxford University Press, London (1934) pp. 199.

31. Einstein, A. and Rosen, N., Phys. Rev. 49 (1936) 404.

32. Hagihara, Y., Jpn. J. Astron. Geophys. 8 (1931) 67.

33. Abrams, L.S., Can. J. Phys. 74 (1996) 701.

\section{Appendix A. Derivation of point-Mass metric}

Substituting (5) into Dingle's expressions (see ref. [29]) for $T_{j}^{i}$ gives

$$
\begin{array}{r}
-8 \pi T_{1}^{1} \equiv-\frac{1}{C}+\frac{C^{\prime 2}}{4 B C^{2}}+\frac{A^{\prime} B^{\prime}}{2 A B C}=0 \\
-8 \pi T_{2}^{2} \equiv \frac{C^{\prime \prime}}{2 B C}+\frac{A^{\prime \prime}}{2 A B}-\frac{C^{2}}{4 B C^{2}}-\frac{B^{\prime} C^{\prime}}{4 B^{2} C}-\frac{A^{\prime 2}}{4 A^{2} B} \\
-\frac{A^{\prime} B^{\prime}}{4 A B^{2}}+\frac{A^{\prime} C^{\prime}}{4 A B C}=0 \\
T_{3}^{3}=T_{2}^{2}=0 \\
-8 \pi T_{4}^{4} \equiv \frac{C^{\prime \prime}}{B C}-\frac{1}{C}-\frac{B^{\prime} C^{\prime}}{2 B^{2} C}-\frac{C^{\prime 2}}{4 B C^{2}}=0
\end{array}
$$

with all other $T_{j}^{i}$ identically zero. (Here and afterwards a superscript prime denotes differentiation with respect to $r$.)

Subtracting (A.1) from (A.4) and multiplying the result by $B C$ (nonzero because of (7)) gives

$$
C^{\prime \prime}-\frac{C^{\prime}}{2}[\ln (A B C)]^{\prime}=0
$$

Since $C^{\prime}=0$ would (in view of (7)) reduce (A.1) to $-1=0$, it follows that $C^{\prime} \neq 0$, hence dividing (A.5) by $C^{\prime}$ gives

$$
\frac{-2 C^{\prime \prime}}{C^{\prime}}-[\ln (A B C)]^{\prime}=0
$$

which integrates at once to

$$
C^{2}=J A B C
$$

with $J$ a constant. Since $A, B$, and $C>0$ and $C^{\prime} \neq 0$, it follows that $J>0$, and thus $C^{\prime}$ never vanishes for $r>0$. 
Solving (A.7) for $B$ and substituting the result into (A.1) gives, after cancelling some nonzero factors,

$$
\frac{-1}{C}+\frac{J A}{4 C}+\frac{J A^{\prime}}{2 C^{\prime}}=0
$$

whence either

$$
\frac{C^{\prime}}{C}=\frac{2 J A^{\prime}}{4-J A} \text { or } A=\frac{4}{J}
$$

The second alternative results in no gravitational force whatever on a distant test particle (as seen from ref. [30], the gravitational acceleration of such a particle in the field of (5) is $-\psi^{\prime} / 2$, where $\psi=A-1$; thus, $A=$ constant would result in zero acceleration), and is therefore ruled out. The first integrates to

$$
C(J A / 4-1)^{2}=K_{0} \equiv \alpha^{2}>0
$$

the positivity of the constant $K_{0}$ being a consequence of that of $C$ and of the non-negativity of $(J A / 4-1)^{2}$. Solving (A.10) for $A$ (and without loss of generality, choosing $\alpha>0)$ gives

$$
A=\frac{4}{J}\left(1 \pm \frac{\alpha}{C^{1 / 2}}\right)
$$

which upon substitution into (A.7) yields

$$
B=\frac{C^{\prime 2}}{4 C\left(1 \pm \alpha / C^{1 / 2}\right)}
$$

Substituting $A$ and $B$ from (A.11) and (A.12) into (A.2) shows that the latter is satisfied identically for arbitrary $C$.

Now, the requirement that (5) be spatially asymptotically flat necessitates that

$$
\frac{C}{r^{2}} \rightarrow 1 \text { as } r \rightarrow \infty
$$

From this and from the fact that $C^{\prime}$ cannot be zero it follows that

$$
C^{\prime}>0 \text { for } r>0
$$

Moreover, from (A.13) and (A.11) we see that

$$
A \rightarrow 4 / J \text { as } r \rightarrow \infty
$$

whence asymptotic flatness requires that $J=4$. This reduces (A.11) to

$$
A=1-\frac{\alpha}{C^{1 / 2}}
$$

the choice of the minus sign being compelled by the fact that the gravitational force on a distant test particle must be attractive (as noted above, the gravitational acceleration of a distant test particle is $-\psi^{\prime} / 2$, where $\psi=A-1= \pm \alpha / C^{1 / 2}$; this acceleration will only be attractive if the lower 
sign is chosen, since both $C$ and $C^{\prime}$ are positive). From (7), (A.14), and (A.16) it follows at once that

$$
C(0+) \geq \alpha^{2}>0
$$

Hence we conclude that the most general triplet satisfying the historical postulates for the point mass is of the form

$$
\begin{gathered}
A=1-\alpha / C^{1 / 2}, \alpha>0 \\
B=\frac{C^{\prime 2}}{4 C\left(1-\alpha / C^{1 / 2}\right)}
\end{gathered}
$$

where $C$ is an analytic function of $r=\left(x^{2}+y^{2}+z^{2}\right)^{1 / 2}$ having the following properties:

$$
\begin{array}{r}
C(0+) \equiv \beta^{2} \geq \alpha^{2}>0 \\
C^{\prime}(r)>0, \text { for } r>0 \\
C(r) / r^{2} \rightarrow 1 \text { as } r \rightarrow \infty
\end{array}
$$

Finally, consider the circle $\gamma(\epsilon): t=t_{0}$ (a constant), $\theta=\pi / 2$, and $r=\epsilon$. Inspection of (10) shows that the proper circumference of $\gamma(\epsilon)$ is $2 \pi[C(\epsilon)]^{1 / 2}$, which by (A.20) tends to $2 \pi \beta \geq 2 \pi \alpha>0$ as $\epsilon \downarrow 0$. Moreover, inspection of (10) and (A.19) shows that the proper radius of $\gamma(\epsilon)$ is

$$
\begin{aligned}
& R_{P}(\epsilon)=\int_{0-}^{\epsilon}[B(r)]^{1 / 2} d r \\
= & \int_{0-}^{\epsilon} \frac{C^{\prime} d r}{2 C^{1 / 2}\left(1-\alpha / C^{1 / 2}\right)^{1 / 2}} \\
= & \int_{\beta}^{[C(\epsilon)]^{1 / 2}}\left[\frac{u}{u-\alpha}\right]^{1 / 2} d u
\end{aligned}
$$

Since $C(0+)$ is finite, and $C(r)$ monotonic and analytic for $r>0$, these last two properties are also true of $[C(\epsilon)]^{1 / 2}$, whence

$$
[C(\epsilon)]^{1 / 2}=\beta+O(\epsilon)
$$

and thus

$$
\begin{aligned}
R_{P}(\epsilon) & =\int_{\beta}^{\beta+O(\epsilon)}\left(\frac{u}{u-\alpha}\right)^{1 / 2} d u \\
& \approx \int_{\beta}^{\beta+O(\epsilon)}\left(\frac{\beta}{u-\alpha}\right)^{1 / 2} d u
\end{aligned}
$$

which for all $\beta$ satisfying (A.20) clearly tends to zero as $\epsilon \downarrow 0$. Consequently, the ratio of the proper circumference to the proper radius of $\gamma(\epsilon)$ does not tend to $2 \pi$ as $\epsilon \downarrow 0$, so that there is a violation of elementary flatness [31] at $r=0$ and $t=t_{0}$, and since $t_{0}$ was arbitrary, at $r=0$ for all $t$. This violation

\footnotetext{
${ }^{6}$ If the boundary $r=0$ in the spatial section is a point, which is the case by virtue of the point mass being located there.
} 
constitutes a "quasiregular singularity" [2] of the space-time associated to $g_{P M}$, and thus we can say that the space-time $\left(M_{0}, g_{P M}\right)$ is analitically inextendible to $r=0$ for all $\beta \geq \alpha$. Moreover, since it is well known [32] that those geodesics of the Hilbert metric $g_{H}$ that do not run into the boundary at $r^{*}=\alpha$ are complete, the diffeomorphism between $g_{H}$ and $g_{P M}$ for any admissible $C$ shows that the same is true of those geodesics of $g_{P M}$ that do not run into the boundary at $r=0$. It follows that the space-times $\left(M_{0}, g_{P M}\right)$ are maximal.

\section{Appendix B. Acceleration scalar}

As shown by Doughty [13], the acceleration of a neutral test particle approaching the point mass along a radial path, as measured by an observer at rest with respect to the point mass, is given by

$$
\begin{array}{r}
a=\frac{\left(-g_{r r}\right)^{1 / 2}\left(-g^{r r}\right)\left|g_{00, r}\right|}{2 g_{00}}=\frac{A^{\prime}}{2 A B^{1 / 2}} \text { for }(5) \\
=\frac{\alpha}{2 C\left(1-\alpha / C^{1 / 2}\right)^{1 / 2}} \text { for }(10) \\
=O\left[1 / 2(\alpha r)^{1 / 2}\right] \text { as } r \rightarrow 0 \text { for }(18)
\end{array}
$$

However, when $R_{P}(\epsilon)$ in (A.23) is evaluated for (18), it is readily found that

$$
R_{P}(r)=O\left[2(\alpha r)^{1 / 2}\right] \text { as } r \rightarrow 0
$$

thus

$$
a \sim 1 / R_{P}(r) \text { as } r \rightarrow 0
$$

which shows that unlike the Newtonian case, a test particle's acceleration tends to infinity inversely as the first power of its proper distance to the central mass.

Lockheed Corporation, 4500 Park Granada Boulevard, Calabasas, CA 91399, U.S.A., and 24345 Crestlawn Street, Woodland Hills, CA 91367, U.S.A. (present address).

\footnotetext{
${ }^{7}$ More precisely, there exists no analytic extension in which $r=0$ corresponds to an interior point in the larger manifold. Any extension in which $r=0$ corresponds to a nonpoint-like locus would alter the character of the point mass and is thus ruled out $a$ priori.
} 